the absence of physical factors. This applies - as in any other branch of medicine-to the decision whether or not to treat by hypnosis.

\section{Consulting Rooms}

Maurice SilvermaN

Beardwood Hospital

Preston New Road

Blackburn BB2 7 BG

\section{References}

MACKETT, J. (1985) Hypnotherapy for subfertility: report of two cases. Proceedings of the British Society of Medical and Dental Hypnosis, 6, 20-21.

Whorwell, P. J., Prior, A. \& Faragher, E. B. (1984) Control trial of hypnotherapy in the treatment of severe refractory irritable bowel syndrome. Lancet, ii, 1232-1234.

- - \& Colgam, S. M. (1987) Hypnotherapy in severe irritable bowel syndrome: further experience. Gut, 28, 423-435.

\section{Reply}

The main aim of my article was to outline the current situation of hypnosis and its possible application in psychiatry, not to discuss details of case reports and possible further application in general practice.

I am sad that Dr Silverman misunderstood my statement of "substitute to treat the untreatable". Hypnotherapy has always been misused and has unfortunately been applied as a main therapy for malignant disorders and even HIV infection which currently cannot be treated by any known conventional methods. Hypnotherapy has also been misused to treat disorders which may be treated by surgery. Therefore, hypnotherapy must not be considered as an alternative or "substitute" or secondline therapy for any disorder which is currently untreatable by conventional or known methods.

I am surprised that Dr Silverman thinks that organic abnormality should not be taken into account as he states in his final paragraph "this may or may not be true". To elaborate the point further Dr Silverman quotes the paper by Mackett (1985) "the use of hypnosis in infertility without any organic basis". How and who decided about the organic basis and why did they not use people with "organic basis"?

I have tried to keep the paper as brief as possible with a view to encouraging psychiatrists in my venture to establish a 'Hypnotherapy Special Interest Group' within the Royal College of Psychiatrists, about which I have already written to the President.

University of Leicester V. M. MatheW Clinical Sciences Building

Leicester Royal Infirmary

PO Box 65

Leicester LE2 7LX
User satisfaction with a psychiatric day hospital

\section{DeAR SIRS}

The Mental Illness Key Area Handbook from the Department of Health emphasises that central to the creation of "appropriate and responsive services" is involvement of users "with the most severe mental illnesses and the most longstanding health and social problems". We wish to report our recent study which examined the pattern of need of patients attending a psychiatric day hospital in an inner city area of high social deprivation.

We used a structured interview on a comprehensive patient sample recruited across all hospital working days and opening hours: the sample (23) was representative of all 96 patients on the attendance register. Day patients tended to be single, middleaged, isolated, with mainly psychotic illness, had an approximately equal sex distribution and ethnic minorities composition similar to the general population. In-patient admissions were about three times the length of other catchment area patients, and social disabilities were severe according to World Health Organisation criteria.

Twenty-one $(91 \%)$ of the patient sample named their key worker. High levels of satisfaction were reported for professionals that patients had most access to and contact with: $74 \%$ of patients rated nurses as "helpful" or "very helpful", while similar ratings for occupational therapists were $96 \%$. Psychiatrists' ratings were lower (consultants: $30 \%$, registrars: $52 \%$ ). The reported helpfulness of psychologists and social workers depended on their accessibility. Average group attendance was ten patients (range 4 to 15); regression analysis showed a consistent level of satisfaction of $71 \%$ across selfselecting groups $(n=15, P<0.0001)$ of all sizes. Most patients $(74 \%)$ believed their mental health was benefitting from attendance, and over half (52\%) said their care was strengthened by sharing it with others.

Our initial, anonymous, self-completed questionnaire (a widely used method) failed to obtain any useful information from this patient population. However, using our structured interview we identified specific areas in which care was subsequently improved. We would stress that in-depth interviewing requires many manhours, including negotiation and consultation with, and feedback to, staff and users. In our opinion, all staff should be involved in planning the audit, and interpretation of the implications for change.

JOAN RUTHERFORD

The Ridgewood Centre

Old Bisley Road, Frimley

Surrey GU16 5QE 